\title{
Safety of users in road evacuation: modelling and DSS for LFA in the planning process
}

\author{
F. Russo \& C. Rindone \\ LAST, Laboratory for Transport Systems Analysis DIMET, \\ Department of Computer Science, Mathematics, Electronics and \\ Transportation, Mediterranea University of Reggio Calabria, Italy
}

\begin{abstract}
In this paper main modelling tools and Decision Support System (DSS) to support evacuation planning in urban system are presented. Project Cycle Management (PCM), integrated with the Logical Framework Approach (LFA), is adopted to link the goals, outcomes, outputs with the strategies and inputs in the internal planning process. Main DSS to support PCM and LFA are presented.

Keywords: planning process, evacuation plan, logical framework approach, software.
\end{abstract}

\section{Introduction}

To plan a system in emergency conditions it is necessary to assess risk and its relative components: probability, vulnerability and exposure [27]. In this paper we analyse evacuation planning to reduce exposure.

Evacuation planning has developed for different systems (single building, land system, transportation system, industrial system) [2, 8, 33]. In recent years, some modelling tools and Decision Support System (DSS) are developed to assist decision makers into prepare evacuation plans [23, 31]. Assessment monitoring and prediction tools are indicated in U.S. guidelines to support an evacuation $[4,6,7]$. U.S. Army Corps of Engineers (USACE) and the Federal Emergency Management Agency (FEMA) have analyzed possible impacts of various hurricane hazards to determine the amount of response time necessary to 
ensure all populations are out of the threatened areas before the effects of any hurricane arrive.

In this paper we recall a proposal of an internal evacuation planning process presented in previous works [24-26]. The process is affected by the objectives to be pursued to implement strategies which respect constraints. Starting from analysis of the present situation, in the plan a set of strategies to adopt for pursuing their objectives is identified. Strategies include a set of interventions that have to evaluate whether objectives could be achieved and constraints respected. Ex ante and ex post evaluations of effects produced by the planned interventions are essential elements of the process [24].

A possible method to evaluate how strategies actually achieve goals, starting from inputs exogenously defined, is the Logical Framework Approach (LFA). The method is a planning tool according to a results-oriented approach $[1,34]$.

LFA is applied in planning by numerous countries. In the United States, the LFA has been applied in the general planning process since 1993 with the Government Performance and Results Act (GPRA) [32]. Many guidelines to implement the LFA are available and are recalled in [22] and [26]. The European Commission adopted LFA as core tool used within Project Cycle Management (PCM). In PCM, a project is defined how a series of activities aimed at bringing about clearly specified objectives within a defined time-period and with a defined budget. PCM is a term used to describe the management activities and decision-making procedures used during the life-cycle of a project (including key tasks, roles and responsibilities, key documents and decision options). PCM, integrated with the LFA, is adopted to allocate and monitor structural funds [3].

A research project called SICURO (riduzione del riSchio medIante proCedure di evacuazione: linee gUida, speRimentazione e sistema di suppOrto alle decisioni) has been developed by LAST (Laboratory for Transport Systems Analysis) with a grant from the European Union and Calabria region: methods and models of the research project can be applied to evaluate ex ante an urban evacuation plan; in the project two experiments are carried out to evaluate ex post the plan.

In this paper, the LFA is considered in relation to evacuation planning of an urban system in emergency conditions. In section 2, USA approach to emergency planning modelling is presented. In section 3, LFA is proposed to transportation planning in evacuation conditions. Methods and models of the SICURO project are applied to calculate ex ante indicators to evaluate an evacuation plan in an urban context. In section 4, an analysis of principal software and DSS that could be used in evacuation planning is presented and an application of LFA in a local evacuation plan is given.

\section{USA approach to emergency planning modelling}

After last events, including the 9/11 terrorist attacks and natural disasters such as hurricanes, USA has produced a relevant advancements in research and in applications relative to emergency planning. UE, enlarged UE, Russia, China, India, Japan and emerging countries have not spent an analogous effort. For this 
reason, in the following, we analyse USA approach to emergency planning modelling.

Emergency planning is a critical focus area of USA general transportation planning process [6]; recommendations and guidance are indicated from the US Department of Homeland Security (DHS); U.S. Department of Transportation's Federal Highway Administration (FHWA) has published a guide focuses on planning and coordination associated with evacuating populations using highways and roads when there is advance notice of an event. American public safety organizations work to improve their capabilities according to the National Response Plan (NRP) and the National Incident Management System (NIMS), which were released by the DHS [4].

DHS promotes Homeland Security Centers of Excellence (HS-Centers) that develop research and education for homeland security solutions. For instance, the Center for Risk and Economic Analysis of Terrorism Events (CREATE), led by the University of Southern California, evaluates the risks, costs and consequences of terrorism, and guides economically viable investments in countermeasures. The National Center for the Study of Preparedness and Catastrophic Event Response (PACER), led by Johns Hopkins University, supports preparedness in the event of a high-consequence natural or man-made disaster, as well as develops guidelines to best alleviate the effects of such an event [9].

DSS and models are developed to support evacuation planning. Abbreviated Transportation Models (ATM) have developed to calculate clearance time in a specific area [20]. The models are based on socioeconomic and behavioural characteristics combined with threat assessment by geographic zone [28]. In Virginia ATM has applied to identify bottlenecks or other problem areas that may develop as a result of evacuation activities. To analyse in depth critical points, micro-simulation software was applied to model the impacts of different storm categories on traffic conditions.

Specific guidelines are published to assist transportation agencies in developing drills and exercises in alignment with the National Incident Management System (NIMS). [...] Guidelines comprise the required elements of a successful transportation emergency exercise program for all modes of transportation. Indications are provided to coordinators at state and local transportation agencies responsible [18].

Exercise programs contribute to improve the cycle between planning, training, exercises, and the identification and implementation of improvement actions. DHS has developed the Homeland Security Exercise and Evaluation Program (HSEEP) to introduce standard for emergency exercises in terms of terminology, common processes and implementation approaches. DHS identifies seven types of exercises whit increasing levels of complexity: discussion based that includes seminars, workshops, tabletops, games; operations based that include drills, functional exercises, and full-scale exercises. HSEEP provides tools and resources to establish self-sustaining exercise programs [10].

The US Department Of Transportation (DOT) has published a literature review about domestic and international evacuation reference materials (plans, 
policies, procedures, ...). DOT reviewed the current guidance for local, State, and Federal agencies regarding evacuations. The review provides a framework of guidance to prepare for and implement a mass evacuation. Documents identify lessons learned and best practices from after-action reports and case studies [5].

\section{Logical framework approach in evacuation planning process}

Internal evacuation planning process can be organised adopting LFA and its two principal stages:

an Analysis Stage, that includes these following steps:

1. Stakeholder Analysis, where individuals or institutions that may directly or indirectly, positively or negatively - affect or be affected by a project or programme is individuated;

2. Problem Analysis where main problems, with cause and effect relationships, are represented whit a Problem Tree;

3. Analysis of Objectives, where the main problems identified at step 2, are translated in goals and outcomes to pursue;

4. Analysis of Strategies, where strategies to pursue objectives are identified.

- a Planning Stage, that includes these following steps:

1. construction of LogFrame matrix, according to Analysis Stage;

2. scheduling of the activities and the resource requirements;

3. determination of the budget.

On adopting LFA, a transportation plan is represented by the following components (Figure 1):

- inputs, needed to implement evacuation procedures, including people (human resources, for instance, police forces, volunteers, coordinators) and available material and immaterial resources;

- activities, that comprise all actions and procedures to prepare and to make an evacuation plan;

- outputs, that comprise products and services performed by the activities;

- outcomes, that represent effects of interventions;

- goals, that represent final results of the plan.

All components are connected by a cause-effect rationale (plan description): starting from available inputs, if activities are implemented then outputs will be delivered; if these outputs are delivered then outcomes will be achieved; if outcomes are achieved then goals will be pursued.

Each component of the plan:

- is measured directly or indirectly by indicators that have to be SMART (Specific, Measurable, Accurate, Realistic and Time bound); for each indicator, a specific target may be assumed;

- is estimated and validated with specific means of verification that give the values of each indicator; 


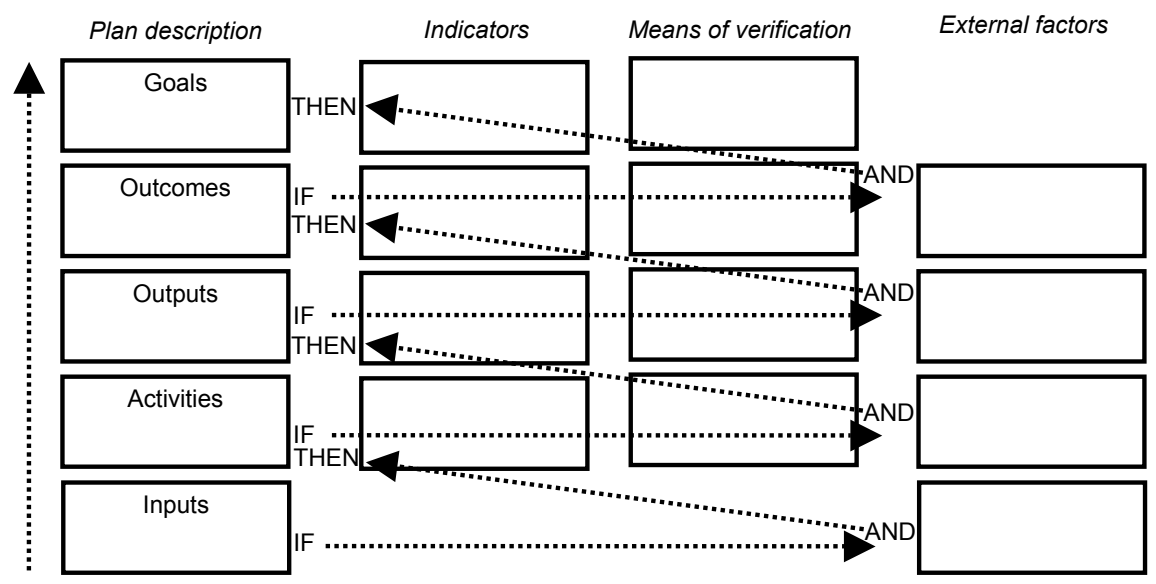

Figure 1: LogFrame of a generic plan.
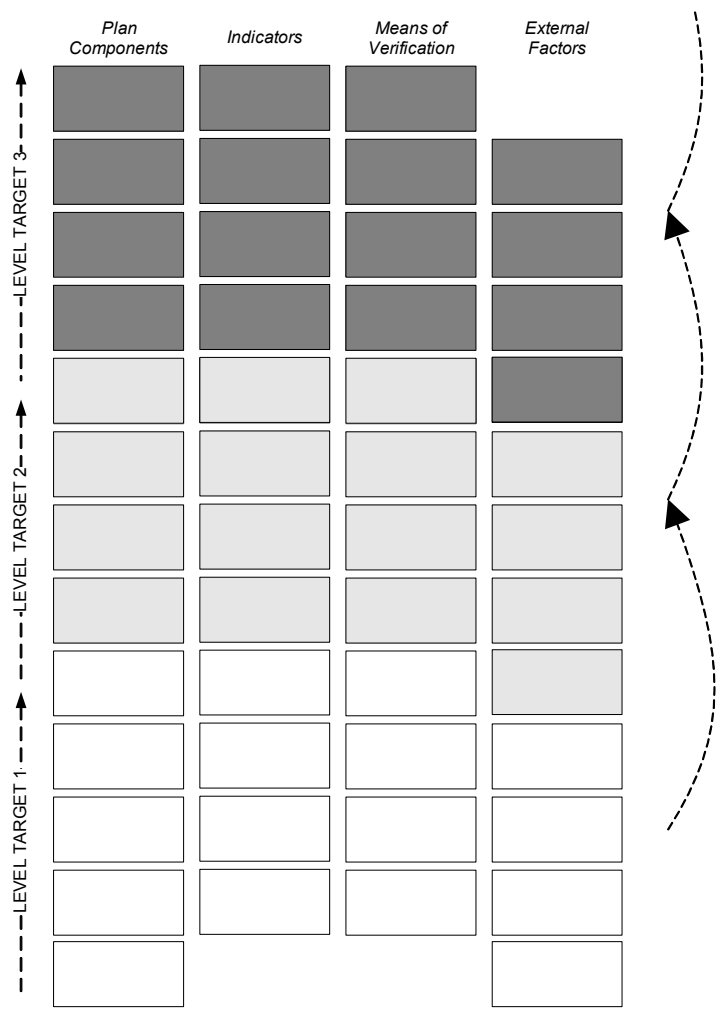

Figure 2: Evolution target's pursuit. 
- may be influenced by external factors, that comprise events, conditions and decisions that can be verified independently by the planner.

The plan is represented by a matrix (LogFrame) constituted by:

- five rows: inputs, activities, outputs, outcomes and goals;

- $\quad$ three columns: indicators, means of verification and external factors.

Specific level of target can be defined, based on inputs that are exogenously defined and constitute external factors (or precondition) to implement the plan. If the level of target to pursuing is higher, a major quantity of inputs is necessary. Starting from new quantity of inputs, all plan components have to modified.

Evolution target's pursuit of depends on inputs expressed as external factors and the pursuit of the goals. The evolution ends when input finish or goals are pursued (Figure 2).

\subsection{Indicators}

The evacuation plan can be evaluated ex ante by means of indicators that can be calculated by applying system of models. In particular:

- $\quad$ possible activity indicators could be expressed in terms of times and number of actions to prepare the plan, other than all the activities developed by technical group;

- possible output indicators could be expressed in terms of number of real evacuation tests conducted;

- $\quad$ possible outcome indicators are expressed in terms of evacuation times;

- $\quad$ possible goal indicators could be expressed in terms of risk reduction.

The same plan can be evaluated ex post by means of real evacuation tests measures, with a monitoring system re-calculating in ex post conditions, the same activity, output, outcome and goal indicators calculated ex ante.

\subsection{Means of verification}

Means of verification of evacuation plan can be represented by methods and models available in literature or Decision Support System (DSS) developed to support evacuation Planning.

There are means of verification for each component of an evacuation plan according to LFA and in particular:

- possible activity means of verification could be database that collects evacuation planning procedures; for instance in USA, Federal Emergency Management Agency (FEMA) has implemented Homeland Security Exercise and Evaluation Program (HSEEP) that collects principal national experiences about evacuation exercise planning [10];

- possible output means of verification could be management tools and reference documents to plan evacuations or exercise; for instance, NEXS System developed in HSEEP program, provides templates and guidance to aid exercise program managers and collects data relative to programmed exercises [10];

- $\quad$ possible outcome means of verification could be methods, models and DSS 
to simulate effects of evacuations or exercises; for instance methods and models resulting from the SICURO project [23] or DSS developed in USA to support evacuation planning;

- possible goal means of verification could be specific for each risk component.

\subsection{External factors}

External factors in the emergency planning process can be represented by a set of elements of uncertainty that characterize the context in which evacuation procedures are activated (for instance meteorological conditions, bad functioning of means of communications, unexpected events).

Each plan components can have external factors that determine effects that can modify the next component.

All plan components depends on available inputs. The input in evacuation planning can be expressed in terms of quantity of material and immaterial available resources to implement evacuation procedures. If the quantity of available inputs is modified, all components, logically connected, have to be reassessed (Figure 2). The iterative process ends when end the starting resources allocated, or when the target relative to goals is obtained.

\subsection{Study case of evacuation planning process}

Methods and models resulting from the SICURO project are applied to verify the Local Civil Protection Plan (LCPP) of Melito Porto Salvo in the province of Reggio Calabria in Italy (study area). By adopting the project guidelines, the plan is tested for a specific event that involves part of the municipal area. The scenario simulated concerns an incident involving a tank transporting hazardous goods which, on a workday morning (8.00 am - 12 noon), is leaking. Starting from the instant in which the presence of a potentially disastrous event is announced, the Mayor decides that the surrounding area must be evacuated.

A subset of results from the SICURO project is reported in [25] and [26], with the estimation of ex ante and the monitoring of ex post indicators of evacuation planning, according to the LFA.

The emergency planning process in the Municipality complies with Italian law [13]. In the Provincial Civil Protection Plan (PPPC) of Reggio Calabria, Melito Porto Salvo is listed as a Mixed Operative Centre. Hence the Municipality draws up a Local Civil Protection Plan (LCPP).

The municipal area covers about 35,300 ha, the urban centre covers about 50ha. In the municipal area there are 10,483 residents and 2,432 workers. After the event, based on information about the type and quantity of hazardous goods, the Mayor identifies an area of about 4.3 ha. In this area, on an average working day morning, 255 residents and 225 employers, teachers and scholars are present.

In the case of the scenario simulated, the Mayor implemented interventions of the LCCP plan for the partial municipal area. 
Two evacuation tests are carried out (I test ; II test). In the I test the town hall and school buildings are involved. In the II test, besides public buildings, private and commercial buildings in the area are involved.

Outcome and goal indicators are evaluated ex ante, applying methods and models resulting from the SICURO. The same indicators are measured ex post during the two evacuation tests and compared with ex ante evaluation.

\section{DSS for LFA}

DSS that integrate analysis of transportation system and project management for planning aren't available in commerce and DSS for planning transportation system with LFA don't exist. Than, in the following, two different typologies of DSS will be analysed.

Standard DSS to support project management in planning are available (section 4.1).

Several commercial DSS could be used to analyse a transportation system in ordinary condition or forced to analyse traffic system in emergency condition. Specific DSS are projected to support emergency planning (section 4.2).

\subsection{DSS for project management in emergency planning}

In recent years some software to support project management and LFA have been developed. Among principal, it's need to refer:

- $\quad$ software to support project management, in general:

- $\quad$ Microsoft Project ${ }^{\circledR}$ (Microsoft, 2007), to represent a plan by a number of components logically linked [16];

- SmartDraw ${ }^{\circledR} \quad$ (SmartDraw.com, 2009) [29] and Microsoft Visio $^{\circledR}$ (Microsoft, 2007), to represent and to analyze a problem using objects that represent, for instance in evacuation modelling, specific events [17];

- Logical Decisions ${ }^{\circledR}$ for Windows, applied in Decision Analysis, to evaluate alternatives of a plan that can be to pursue specific objectives or goals; alternatives are represented with quantitative or qualitative measures; in the tool it's possible to define a goals hierarchy, a structure that links the evaluation measures to overall goal; goals are used to combine and link the measures [15];

- $\quad$ software to support Project Cycle Management: Project Facilitator ${ }^{\circledR}$ (Live Application Technology, 2004) is an online software system; the tool supports both monitoring and evaluation functions [21] (Figure 3);

- $\quad$ software to support Logical Framework Approach: LogFrame for Windows, vers. 1.0 (Maizemoor International, 2008) to construct a LogFrame specifying external factors that could influences in achieving goals of the plan [14] (Figure 4);

- $\quad$ software to support Project Cycle Management and Logical Framework Approach TeamUP-PCM ${ }^{\circledR}$ (TEAM Technologies, Inc., 2008), including stakeholder problem analysis, results planning, alternative selection, project 
design, implementation planning, progress monitoring; it is possible to implement the LogFrame [30].

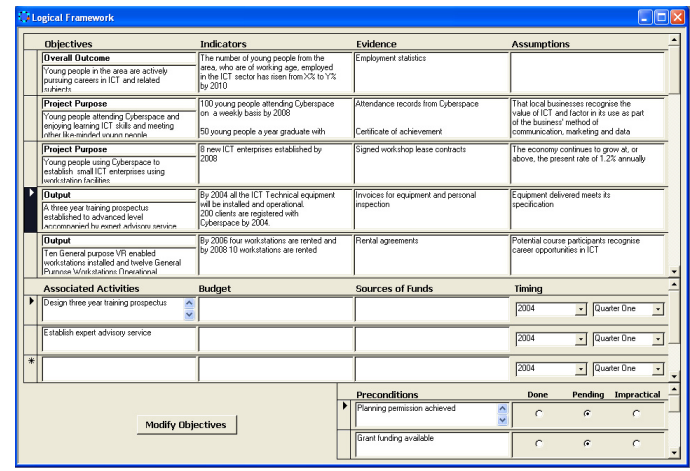

Figure 3: $\quad$ Project Facilitator $^{\circledR}$, vers. 1.4.

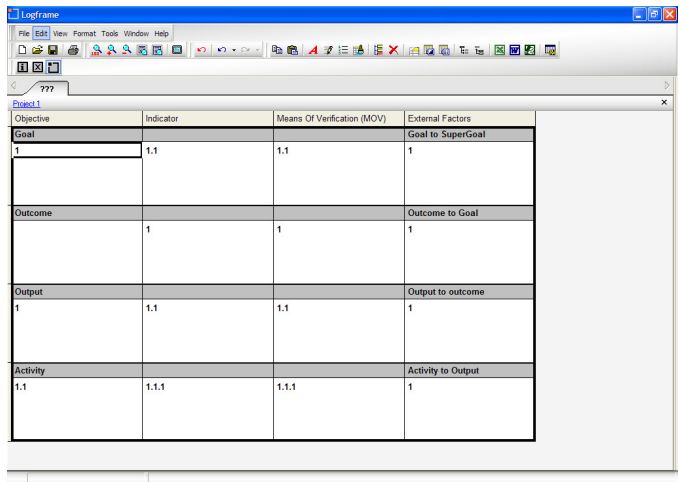

Figure 4: $\quad$ LogFrame for Windows, vers. 1.0.

\subsection{DSS for emergency planning}

Different commercial DSS are available to analyse transportation system in ordinary condition. Among principal we can recall: EMME/2, TransCAD, VISUM, CUBE. Some of these software can be adapted to analyse transportation system in emergency condition. More specifically, micro simulation programs can be used to analyse traffic system in evacuation planning. One example is Dynasmart-X, which provides estimates of network traffic conditions, predictions of network flow patterns over the near and medium terms in relation to different interventions. Another example is CORSIM, that is a microscopic traffic simulator of traffic and traffic control systems using commonly accepted vehicle and driver behavior models. CORSIM combines two of the most widely used traffic simulation models, NETSIM for surface streets, and FRESIM for freeways. 
Specific software is designed to support emergency planning or single component of a plan (activity, output, outcome or goal) . For instance: MASSVAC (MASS eVACuation) is a simulation model of highway networks to calculate evacuation time; OREMS (Oak Ridge Evacuation Modeling System), is a probabilistic model for a systems approach to evacuation operations; ETIS (Evacuation Traffic Information System) is a tool to monitor highway evacuation processes, to share information during an evacuation to help decision makers make adjustments in their evacuation routing [4]; HURREVAC (HURRicane EVACuation) is an hurricane evacuation decision-making tools that estimate clearance times to help determine when and if evacuations should begin [12]; SLOSH Model (Sea, Lake, and Overland Surges from Hurricanes) that allows to predict specific event (e.g. storm surge) [4]; HAZUS-MH (Multi-Hazards U.S. Software) is a tool to predict the impacts of disasters to buildings and infrastructures (bridges, galleries, ...) from various hazards; CATS (Consequence Assessment Tool Set) / Joint Assessment of Catastrophic Events (JACE) is a tool to estimate damage and assists emergency managers to develop mitigation strategies [4]; MitigationPlan.com System to identify hazards that could impact their community and the need for an evacuation; Abbreviated Transportation Models (ATM) to estimate the number of people and vehicles that will be evacuating each jurisdiction under various storm scenarios [19].

\section{References}

[1] Canadian International Development Agency - CIDA (1997), "CIDA's Business Process RoadMap. Overview" http://www.acdicida.gc.ca/CIDAWEB/acdicida.nsf /En/JUD-131105815-LQY (October 2007)

[2] Cantarella G. E., Cepollina E., Delfino G., Di Gangi M., Pratelli A., Rindone C., Russo F., Vitetta A. (2005), Linee guida per la redazione dei piani di evacuazione mediante la simulazione dei sistemi di trasporto in condizioni di emergenza Laruffa, Reggio Calabria, Italy

[3] European Commission (2004), "Project Cycle Management" http://ec.europa.eu/europeaid/multimedia/publications/publications/manuals -tools/ t101_en.htm (February 2009)

[4] Federal Highway Administration (2006), Using Highways during Evacuation operations for events with advance notice, U.S. Department of Transportation, http://www.ops.fhwa.dot.gov/publications/evac_primer/00_evac_primer.ht m (July 2008)

[5] Federal Highway Administration, Emergency Transportation Operations http://www.ops.fhwa.dot.gov/publications/publications.htm\#eto (February 2009)

[6] Federal Highway Administration, Federal Transit Administration (2007), The Transportation Planning Process Key Issues, Publication of the Transportation Planning Capacity Building Program, http://www.planning.dot.gov/documents/briefingbook/ bbook.htm 
[7] Goldblatt R. B., Weinisch, K. (2008), "Evacuation Planning, Human Factors, and Traffic Engineering: Developing Systems for Training and Effective Response" TR News, Issue Number: 238, pp 13-17, Transportation Research Board, Washington, DC

[8] Han L.D., Yuan F., Urbanik T. (2007), "What is an effective evacuation operation?" Journal Of Urban Planning and Development, pp 3-8, ASCE

[9] Homeland Security Centers of Excellence, http:// www.dhs.gov /xres/ programs/ editorial_0498.shtm (February 2009)

[10] Homeland Security Exercise and Evaluation Program (HSEEP), https://hseep.dhs.gov/pages/1001_HSEEP7.aspx (February 2009)

[11] http://www.nae.usace.army.mil/projects/ma/hurricanemaps/mainesumreport .pdf

[12] HURRicane EVACuation program, www.hurrevac.com (February 2009)

[13] Law no. 225 of 24 February 1992, Istituzione del Servizio nazionale della protezione civile (Gazz. Uff., 17 March, no. 64, Suppl. ordinario, Italy, 1992).

[14] LogFrame for Windows, http://www.maizemoor.com/pages/2/index.htm (February 2009)

[15] Logical Decisions, http://www.logicaldecisions.com/ (February 2009)

[16] Microsoft Office Project 2007, http://office.microsoft.com/itit/project/default.aspx (February 2009)

[17] Microsoft Office Visio 2007, http://office.microsoft.com/it-it/visio /FX100487861040.aspx (February 2009)

[18] National Research Council (U.S.), American Association of State Highway and Transportation Officials, National Cooperative Highway Research Program, Transit Cooperative Research Program, Transit Development Corporation, McCormick Taylor (2006) "Transportation Security: Guidelines for Transportation Emergency Training Exercises" Transportation Research Board, ISBN 0309098505 (0-309-09850-5), http:// www.trb.org /news/ blurb_detail.asp?id $=6007$ (February 2009)

[19] PBS\&J, http://www.pbsju.com/Press/Highlights/Summer2007/6.asp (February 2009)

[20] Post, Buckley, Schuh and Jernigan, Inc. (2007), "Maine Hurricane Evacuation Study Transportation Analysis - 2007"

[21] Project Facilitator, http://www.locallivelihoods.com/ProductsProjectFacilitator.htm (February 2009)

[22] Rindone C. (2007), "Logical Framework Approach nella pianificazione dei trasporti: uno stato dell'arte", Proceedings of XXVIII Italian Conference of Regional Science, Bolzano, Italy

[23] Russo F. (2007), Urban Transport: Safety of Users in Road Evacuation, WIT Press

[24] Russo F., Rindone C. (2007), Dalla pianificazione alla progettazione dei sistemi di trasporto: processi e prodotti, Franco Angeli, Milan, Italy 
[25] Russo F., Rindone C., (2007), "Safety of users in road evacuation: planning internal processes and guidelines", in Urban Transport: Safety of Users in Road Evacuation, Russo F. (ed.), WIT Press

[26] Russo F., Rindone C., (2008), "Safety of users in road evacuation: the logical framework approach in evacuation planning", in Urban Transport: Safety of Users in Road Evacuation, Russo F. (ed.), WIT Press

[27] Russo F., Vitetta A. (2006), "Risk evaluation in a transportation system", International Journal of Sustainable Development and Planning, Vol. 1, Issue 2

[28] Shen Y., Sokolowski J. A. (2007) "Hampton Roads hurricane evacuation arterial routes preliminary study" Maurice J. Ades (Ed.), Proceedings of the 2007 Spring Simulation Multiconference, SpringSim 2007, Norfolk, Virginia, USA, March 25-29, 2007, Volume 3. SCS/ACM 2007, ISBN 156555-314-4

[29] SmartDraw, http://www.smartdraw.com/ (February 2009)

[30] TEAM Technologies, Inc., http://www.teamusa.com/ (February 2009)

[31] Wei H., Zeng Q., Hu H., Wang X., Kukreti A. (2007), "Integrated Urban Evacuation Planning Framework for Responding to Disasters over Surface Transportation Network" in TRB 87th Annual Meeting Compendium of Papers DVD, Washington, DC

[32] White House, Office of Management and Budget (1993), “Government Performance Results Act of 1993", http://www.whitehouse.gov/omb/mgmtgpra/gplaw2m.html\#h1 (October 2007)

[33] White R. A., Blumenberg E., Brown K. A., Contestabile J. M., Haghani A., Howitt A. M., Lambert T. C., Morrow B. H., Setzer M. H., Stanley E. M., Velásquez III A., Humphrey N. P. (2008), Special Report 294: The Role of Transit in Emergency Evacuation, Transportation Research Board, Washington, DC, www.TRB.org (July 2008)

[34] World Bank (2000), "The LogFrame Handbook: A logical framework approach to project cycle management", Washington, DC: World Bank, (October 2007) 